\title{
5-Fluorouracil attenuates dextran sodium sulfate-induced acute colitis in mice
}

\author{
JUNHUA XIAO ${ }^{1 *}$, ZHANJUN LU $^{2}$, JIAQING SHENG ${ }^{3}$, YUNNA SONG $^{2}$, \\ WEILIANG JIANG ${ }^{2}$, FEI LIU ${ }^{1 *}$ and PING ZHENG ${ }^{1 *}$ \\ ${ }^{1}$ Department of Gastroenterology, Shanghai East Hospital, Tongji University, Shanghai 200092 \\ ${ }^{2}$ Department of Gastroenterology, The First People's Hospital, Shanghai Jiao Tong University, Shanghai 200080; \\ ${ }^{3}$ Department of Gastroenterology, The First Affiliated Hospital of Soochow University, Suzhou, Jiangsu 215006, P.R. China
}

Received April 8, 2015; Accepted January 6, 2016

DOI: $10.3892 / \mathrm{mmr} .2016 .4858$

\begin{abstract}
Fluorouracil (5-FU) has been predominantly used in the clinic for cancer chemotherapy. Previous studies have demonstrated that 5-FU has an anti-inflammatory function. In the current study, the potential therapeutic role of 5 -FU in dextran sodium sulfate (DSS)-induced acute mouse colitis was investigated. Effects on the severity of colitis were studied via histochemical and immunohistochemical staining, cytokine levels were determined by reverse transcriptoin-quantitative polymerase chain reaction and the effect of 5-FU on NF- $\mathrm{BB}$ was examined by western blotting. Administration of 5-FU ameliorated the severity of acute DSS-induced colitis. The disease activity score was significantly lower in the 5-FU + DSS-treated mice compared with the DSS-treated group $(\mathrm{P}<0.01)$. Tumor necrosis factor- $\alpha$, interleukin-1 $\beta$ and interferon $\gamma$ mRNA expression levels were significantly downregulated in the colon tissue of DSS mice treated with 5-FU compared with the untreated DSS mice $(\mathrm{P}<0.05)$. In addition, the number of $\mathrm{CD}^{+} \mathrm{T}$ cells in the colonic lamina propria and myeloperoxidase activity were significantly decreased in the 5-FU + DSS-treated mice $(\mathrm{P}<0.05)$. Furthermore, 5-FU treatment significantly reduced $\mathrm{p}-\mathrm{NF}-\kappa \mathrm{B}-\mathrm{p} 56$ protein expression levels in the colon tissue of DSS-treated mice $(\mathrm{P}<0.05)$. The present results demonstrated that 5-FU minimizes the abnormal immune cytokine response and relieves the pathophysiological disorders associated with experimental acute colitis. Thus, the modulating
\end{abstract}

Correspondence to: Dr Fei Liu or Dr Ping Zheng, Department of Gastroenterology, Shanghai East Hospital, Tongji University, 150 Jimo Road, Pudong New District, Shanghai 200092, P.R. China E-mail: liufeiguo2010@163.com

E-mail: zhengpingdoctor@126.com

${ }^{*}$ Contributed equally

Key words: 5-fluorouracil, inflammatory bowel disease, colitis, anti-inflammation, nuclear factor- $\kappa \mathrm{B}$ inflammatory response role of 5-FU may be partially associated with inhibiting $\mathrm{NF}-\kappa \mathrm{B}$ activation and 5-FU may be a novel therapeutic strategy for the treatment of inflammatory bowel disease.

\section{Introduction}

Inflammatory bowel disease (IBD) is an umbrella term for a range of diseases, of which ulcerative colitis (UC) and Crohn's disease (CD) are the two prevailing entities, and is characterized by chronic inflammation of the colon (1). Patients with IBD have intermittent disease flare-ups interspersed with periods of remission (2). The highest reported prevalence values for IBD are in Europe (UC, 505/100,000 persons; CD, 322/100,000 persons) and North America (UC, 249/ 100,000 persons; CD, 319/100,000 persons) (3). The life-time risk of colorectal cancer (CRC) in a patient with IBD is 2-4 times greater than the risk of the control population, which is $\sim 5 \%$ (4). IBD compromises the quality of life of patients (5) and significantly increases the risk of developing CRC compared with the general population (6). The current treatment for IBD remains unsatisfactory and utilizes drugs, such as mesalamine, glucocorticoids, azathioprine and anti-tumor necrosis factor (TNF- $\alpha$ ) agents (including infliximab and adalimumab). These agents are not universally effective, and their use is further restricted by the occurrence of side effects, including bone marrow suppression, pancreatitis, opportunistic infections and malignancies $(7,8)$. Therefore, there is a requirement for the identification of novel immunomodulators for remission induction or maintenance in patients with IBD.

5-Fluorouracil (5-FU) is a widely used anticancer agent, whose anti-tumor mechanism remains unclear; however it appears to interfere with the DNA synthesis and mRNA translation (9). In addition, 5-FU has been reported to inhibit protein synthesis and secretion (10). Thus, investigations regarding 5-FU primarily focus on the potential treatment of various types of cancer (11). Furthermore, the role of 5-FU in intestinal physiology, and response to injury and inflammation in vivo remain poorly defined. Notably, a previous study suggested that 5-FU may decrease abnormal immune cytokine responses, and thus relieve pathophysiological disorders, through its anti-metabolic and immunosuppressive effect in a model of 
acute pancreatitis (12). In the present study, we hypothesize that 5-FU exhibits an anti-inflammatory effect in colitis.

In order to investigate the possible anti-inflammatory effect of 5-FU treatment and the mechanisms underlying it in colitis, a dextran sodium sulfate (DSS)-induced mouse model of colitis was utilized for experimental purposes.

\section{Materials and methods}

Induction of colitis and treatment. Female BALB/c mice, aged 6-8 weeks and weighing $20 \mathrm{~g}$, were purchased from Shanghai SLAC Laboratory Animal Co., Ltd., (Shanghai, China) and maintained at $22^{\circ} \mathrm{C}$ with a 12 -h light/dark cycle and ad libitum access to water and a standard rodent diet. Mice were randomly divided into four groups: Normal control, DSS-treated, 5-FU-treated and DSS + 5-FU-treated groups ( $n=20$ per group). Colitis was induced in the mice with $4 \%$ DSS (molecular weight, 36-50 kDa; MP Biomedicals, LLC, Santa Ana, CA, USA) dissolved in tap water administered ad libitum (days 1-7). Control mice were administered tap water ad libitum (days 1-7). 5-FU (Sigma-Aldrich, St. Louis, MO, USA) was administered intraperitoneally at a dosage of $15 \mathrm{mg} / \mathrm{kg}$ body weight once daily for the first 3 days of DSS treatment. Control mice received phosphate-buffered saline (PBS; Sangon Biotech Co., Ltd., Shanghai, China) via intraperitoneal injection once daily for the first three days, at the same volume of 5-FU. Half the mice from each group were sacrificed via intraperitoneal injection of $4 \%$ pentobarbital on day 3 and the other half on day 7. Colon tissue was excised and fixed in $4 \%$ paraformaldehyde (Sangon Biotech Co., Ltd.), or frozen in liquid nitrogen and stored at $-80^{\circ} \mathrm{C}$. The Animal Welfare Committee of Shanghai East Hospital approved all experimental procedures.

Clinical assessment. Multiple clinical parameters were measured throughout the present study, including the percentage of initial weight, stool consistency and fecal bleeding. These were recorded and graded for severity on a scale between 0 (less severe) and 3 (most severe), as previously described (13). In order to calculate the disease activity score, each parameter was then rescored on a scale between 0 and 3. Final scoring was as follows: Percentage of initial weight $(0,>99 \% ; 1,92-99 \% ; 2,85-91 \%$; and $3,<85 \%)$; stool consistency ( 0 , normal; 1 , slightly soft; 2 , loose; and 3 , liquid); bleeding on hemoccult test ( 0 , negative; 1 , faint blue; 2 , blue; 3 , red) (Sangon Biotech Co., Ltd.). The mean of these parameters represented the disease activity score.

Gross and histological analysis. Inflammation-induced reduction in colon length was used as a marker for the severity of acute DSS-induced inflammation of the colon (14). Half the mice from each group were sacrificed on day 3 and the other half on day 7. For all mice, the entire colon was removed and measured following sacrifice. The extracted colon tissue was spread on a plastic sheet, fixed with $4 \%$ paraformaldehyde and embedded in a paraffin block. Sections of paraffin-embedded tissue (4- $\mu$ m-thick) were subjected to hematoxylin and eosin (H\&E; Beyotime Institute of Biotechnology, Shanghai, China) staining for the evaluation of colitis severity. Histological sections were scored for severity based on the following parameters (ranging between 0 and 3): Crypt damage; leucocyte infiltration; and submucosal edema and haemorrhage. The score was further multiplied by the extent of involvement (x1, <10\%; x2, 10-25\%; x3, >25\%). A value of four was added to the histological score if there was evidence of transmural involvement (15). The scoring was graded in a blinded manner by two independent investigators.

Reverse transcription-quantitative polymerase chain reaction (RT-qPCR). Total RNA was extracted from the colon tissue of mice with induced colitis using Tripure Isolation Reagent (Roche Diagnostics, Basel, Switzerland) and RNA quality was assessed by the A260/A280 ratio using a BioPhotometer (Eppendorf, Hamburg, Germany). Total RNA (1 $\mu \mathrm{g})$ was reverse transcribed using a Prime Script RT Reagent kit with gDNA Eraser (Perfect Real Time; Takara Bio, Inc., Otsu, Shiga, Japan). RT-qPCR was conducted using the SYBR Green Master Mix kit (Takara Biotechnology Co., Ltd., Dalian, China), according to the manufacturer's protocol. The relative quantity of target mRNA was determined using the quantification cycle $(\mathrm{Cq})$ method (16) by normalizing target mRNA Cq values to those for $\beta$-actin. The primer sequences used were as follows: TNF- $\alpha$, F 5'-GTCGTAGCAAACCACCAAGTG-3' and R 5'-CAGATTTGTGTTGGTCCTTC-3'; interleukin-1 $\beta$ (IL-1 $\beta$ ), F 5'-AGGCTGCTCTGGGATTC-3' and R 5'-GCC ACAACAACTGACGC-3'; interferon $\gamma$ (IFN- $\gamma)$, F 5'-TGT AGTGAGGAACAAGCCAGAG-3' and R 5'-TACATTTGC CGAAGAGCC-3'; IL-10, F 5'-ATGCTGCCTGCTCTTACT GAC-3' and R 5'-CGGTTAGCAGTATGTTGTCCAG-3'; and $\beta$-actin, F 5'-GTCCACCTTCCAGCAGATGT-3' and R 5'-AGG GAGACCAAAGCCTTCAT-3'. RT-qPCR was performed on an Applied Biosystems Step One Real-Time PCR System (Thermo Fisher Scientific, Inc., Waltham, MA, USA).

Myeloperoxidase (MPO) assay. Neutrophil infiltration in the colon was monitored by measuring the MPO activity (17). Briefly, segments of colon were homogenized in PBS at $50 \mathrm{mg} / \mathrm{ml}$ (50 mmol/1, pH 6.0) with $0.5 \%$ hexadecyltrimethylammonium bromide (Sangon Biotech Co., Ltd.). Samples were frozen and thawed three times, and then centrifuged at $30,000 \mathrm{x} \mathrm{g}$ for $20 \mathrm{~min}$ for $4^{\circ} \mathrm{C}$. The supernatants were diluted 1:30 with assay buffer consisting of PBS, $0.167 \mathrm{mg} / \mathrm{ml}$ o-dianisidine (Sigma-Aldrich) and $0.0005 \% \mathrm{H}_{2} \mathrm{O}_{2}$ (Sangon Biotech Co., Ltd.). The colorimetric reaction was measured using a Nanodrop 2000 spectrophotometer (Thermo Fisher Scientific, Inc.). MPO activity was calculated as follows: MPO activity $=(\mathrm{A} 450 \times 13.5) /$ tissue weight $(\mathrm{g})$, where $\mathrm{A} 450$ is the change in the absorbance at a wavelength of $450 \mathrm{~nm}$ between 1 and $3 \mathrm{~min}$ after the initiation of the reaction. The coefficient 13.5 was determined empirically for $1 \mathrm{U}$ MPO activity to represent the amount of enzyme that will reduce $1 \mathrm{~mol}$ peroxide/min.

Immunohistochemistry. For immunohistochemical staining, slides were deparaffinized in xylene (Sinopharm Chemical Reagent Co., Ltd., Shanghai, China), rehydrated and immersed in $1.5 \% \mathrm{H}_{2} \mathrm{O}_{2}$ in PBS for $30 \mathrm{~min}$. Slides were then incubated for $1 \mathrm{~h}$ with horseradish peroxidase-conjugated avidin-biotin complex. Subsequently, slides were incubated overnight at $4^{\circ} \mathrm{C}$ with rat anti-mouse CD4 (14-9766; eBioscience, Inc., 
San Diego, CA, USA) or rabbit anti-mouse MPO (ab9535; Abcam, Cambridge, UK) primary antibodies diluted 1:50 in PBS containing 5\% bovine serum albumin (BSA; Sangon Biotech Co., Ltd.) and 10\% goat serum (Thermo Fisher Scientific, Inc.). Following washing three times with PBS for $5 \mathrm{~min}$, biotinylated secondary goat anti-rabbit (1:100; A0277; Beyotime Institute of Biotechnology) or rabbit anti-rat (1:100; ab6733; Abcam) polyclonal antibodies were added to the sections and incubated at room temperature for $1 \mathrm{~h}$. Streptavidin-horseradish peroxidase was then added and, following a 40-min incubation, the sections were stained with 3,3'-diaminobenzidine from a ChemMate and EnVision detection kit (Gene Tech Biotechnology Co., Ltd., Shanghai, China) and counterstained with hematoxylin. $\mathrm{CD} 4^{+} \mathrm{T}$ cells were counted in a blinded manner in 10 intercrypt spaces per mouse using an Olympus BX41-32P02-FLB3 microscope (Olympus Corporation, Tokyo, Japan).

Western blot analysis. Colon tissue was collected, immediately placed in liquid nitrogen and pulverized with a mortar. Tissue was homogenized using lysis buffer $(50 \mathrm{mM}$ Tris- $\mathrm{HCl}$, pH 7.6; $150 \mathrm{mM} \mathrm{NaCl} ; 1$ mM EDTA; $1 \%$ (m/v) NP-40; $0.2 \mathrm{mM}$ phenylmethylsulfonyl fluoride; $0.1 \mathrm{mM} \mathrm{NaF}$; and $1.0 \mathrm{mM}$ dithiothreitol). Whole cell extracts were prepared by lysing the cells in cold radioimmunoprecipitation assay buffer containing a mixture of proteasome inhibitors (Beyotime Institute of Biotechnology). Lysates were then centrifuged at $15,100 \mathrm{x} \mathrm{g}$ for $20 \mathrm{~min}$ at $48^{\circ} \mathrm{C}$, and the supernatant was collected. The homogenate was centrifuged at $48^{\circ} \mathrm{C}$ for $15 \mathrm{~min}$ at $13,000 \mathrm{x} \mathrm{g}$, and the supernatant was then collected. The concentration of proteins was detected using a BCA assay with a Varioskan Lux Multimode Microplate Reader (Thermo Fisher Scientific, Inc.). Protein samples were separated by sodium dodecyl sulfate-polyacrylamide gel electrophoresis at $80 \mathrm{~V}$ for $2 \mathrm{~h}$ at room temperature and then transferred to nitrocellulose membranes (EMD Millipore, Billerica, MA, USA), which were blocked with $1 \%$ BSA in PBS for $1 \mathrm{~h}$ at $37^{\circ} \mathrm{C}$. The blots were incubated with specific primary antibodies overnight at $48^{\circ} \mathrm{C}$, followed by an IRDye 800 -conjugated secondary antibody for $1 \mathrm{~h}$ at $37^{\circ} \mathrm{C}$. Protein expression was detected using the Odyssey Infrared Imaging System (LI-COR, Inc., Lincoln, NE, USA). All blots were stripped and reprobed with polyclonal $\beta$-actin antibody to ascertain equal loading of proteins. Densitometric analysis was performed using Quantity One software (Bio-Rad Laboratories, Inc., Hercules, CA, USA). Briefly, the linear range was detected and the background was subtracted prior to normalization to $\beta$-actin, which was considered to be 1 . Once normalized values were determined for each replicate, the respective means, P-values and fold changes were calculated.

Statistical analysis. Data are expressed as the mean \pm standard error of the mean. Statistical significance of differences between treatment and control groups was determined by Student's t-test. Data were analyzed with one-way analysis of variance, followed by Student's t-test for experiments involving 2 groups or Dunnett's t-test for experiments involving $>2$ groups. Statistical analyses were performed using SPSS 19.0 (IBM SPSS, Armonk, NY, USA). $\mathrm{P}<0.05$ was considered to indicate a statistically significant difference.
A


D



Figure 1.5-FU attenuates the severity of acute DSS-induced colitis.5-FU-treated mice displayed (A) less weight loss, (B) less bleeding and (C) greater stool consistency compared with the DSS-treated group. (D) 5-FU-treated mice had lower disease activity scores, most pronounced at day $7(\mathrm{n}=10)$. ${ }^{*} \mathrm{P}<0.05$ ${ }^{* *} \mathrm{P}<0.01$ for $5-\mathrm{FU}+\mathrm{DSS}$ vs. NC. ${ }^{\#} \mathrm{P}<0.05,{ }^{\# \#} \mathrm{P}<0.01$ for DSS vs. NC. NC, normal control; DSS, dextran sodium sulfate; 5-FU, 5-fluorouracil.

\section{Results}

Administration of 5-FU attenuates the severity of acute DSS-induced colitis. The effect of 5-FU on the severity of acute DSS-induced colitis was assessed. Mice were treated with 5-FU or PBS with the administration of $4 \%$ DSS in 
A



C

Day 3




$+$


$-$





$+$

Figure 2.5-FU treatment (A) increases the length of colon and (B) improves the histopathological score of colitis on day 7 compared with the DSS-treated mice. (C) Microscopic appearance of representative hematoxylin and eosin stained sections of colon in control, DSS-treated, 5-FU-treated and DSS + 5-FU-treated mice at days 3 and 7 . All ten mice per group were scored and one section is displayed as representative of that group (magnification, $\mathrm{x} 100)$. ${ }^{\mathrm{P}<0.05 .} \mathrm{NC}$, normal control; DSS, dextran sodium sulfate; 5-FU, 5-fluorouracil.

the drinking water. 5-FU + DSS-treated mice experienced significantly less weight loss $(\mathrm{P}<0.05$; Fig. $1 \mathrm{~A})$, bleeding $(\mathrm{P}<0.05$; Fig. 1B) and loss of stool consistency $(\mathrm{P}<0.05$; Fig. 1C) compared with the DSS-treated mice. These clinical markers suggest reduced DSS-induced inflammatory changes in the 5-FU-treated mice. Consequently, the disease activity score was significantly lower in the 5-FU + DSS-treated mice compared with the DSS group $(\mathrm{P}<0.05)$, particularly at day 7 $(\mathrm{P}<0.01$; Fig. 1D).

In accordance with the clinical data, gross and histological parameters, including crypt damage, leucocyte infiltration, colon length and histological score, favored the 5-FU + DSS-treated mice, suggesting less severe disease compared with the DSS-treated mice. Accordingly, the mean length of colon in the 5-FU + DSS-treated mice $(8.35 \pm 1.23 \mathrm{~cm})$ was significantly longer than in the control group $(6.48 \pm 0.89 \mathrm{~cm}$; $\mathrm{P}<0.05$; Fig. $2 \mathrm{~A})$, suggesting decreased inflammation. In addition, the mean histological score from colon tissue in the 5-FU group was significantly lower than the DSS group $(\mathrm{P}<0.05$; Fig. $2 \mathrm{~B})$. Fig. $2 \mathrm{C}$ demonstrates that 5-FU treatment prevented the abnormal architecture change in the colon that occurred following DSS treatment. The 5-FU control group did not indicate a distorted colon architecture (Fig. 2C). Furthermore, the dose of $15 \mathrm{mg} / \mathrm{kg} \mathrm{5-FU} \mathrm{resulted} \mathrm{in}$ no significant histological or biochemical damage to the liver and kidney of the mice compared with the DSS-treated group (Fig. 3).

5-FU decreases the levels of proinflammatory cytokines in the colon of DSS-induced mice at day 7. Oral administration of DSS is toxic to the colonic epithelium and triggers inflammation by disrupting the compartmentalization of commensal bacteria in the gut with high levels of proinflammatory cytokines, such as TNF- $\alpha$, IL- $1 \beta$ and IFN- $\gamma(18,19)$. The release of cytokines is considered to be an indicator of the inflammatory response. Thus, the mRNA expression levels of TNF- $\alpha$, IL-1 $\beta$ and IFN- $\gamma$ in the colon tissue were detected using RT-qPCR. As demonstrated in Fig. 4, treatment with DSS increased the release of TNF- $\alpha$, IL- $1 \beta$ and IFN- $\gamma$ in mice compared with the normal control group. Notably, in mice treated with 5-FU, the DSS-induced secretion of TNF- $\alpha(\mathrm{P}<0.05)$, IL-1 $\beta(\mathrm{P}<0.01)$ and IFN- $\gamma(\mathrm{P}<0.05)$ were significantly reduced, indicating that 5-FU may inhibit inflammation in intestinal epithelial cells. The expression levels of the anti-inflammatory cytokine IL-10 did not significantly change.

5-FU reduces the expression levels of MPO and the number of $\mathrm{CD}^{+}$cells in the intestinal mucosal of DSS-induced mice at day 7. Another trait of colitis is the invasion of immune cells to the intestinal mucosal with increased expression levels of 
A



B



D



C



$\mathbf{E}$



Figure 3. Effect of 5-FU (15 mg/kg) on histological and biochemical changes in liver and kidney tissue of mice. (A) Representative pictures of hematoxylin and eosin staining (magnification, $x$ 400). Change of (B) ALT, (C) AST, (D) creatinine and (E) urea in control, DSS-treated, 5-FU-treated and DSS + 5-FU-treated mice. Data are expressed as the mean \pm standard error of the mean $(n=10)$. NC, normal control; DSS, dextran sodium sulfate; 5-FU, 5-fluorouracil; ALT, alanine transaminase; AST, aspartate aminotransferase.



Figure 4. Effect of 5-FU treatment on the production of pro-inflammatory cytokines in mice with DSS-induced colitis. Measurement of mRNA levels of secreted cytokines from colon segments of mice treated at day 7. Data are expressed as the mean \pm standard error of the mean $(\mathrm{n}=10) .{ }^{*} \mathrm{P}<0.05$ and ${ }^{* *} \mathrm{P}<0.01$ vs. the DSS group. NC, normal control; DSS, dextran sodium sulphate; 5-FU, 5-fluorouracil; IL, interleukin; TNF- $\alpha$, tumor necrosis factor- $\alpha$; IFN- $\gamma$, interferon- $\gamma$.
MPO and CD4 (20). There was a marked increase in lymphocytic infiltration and loss of glandular architecture in the DSS-treated group compared with the 5-FU + DSS-treated group (Fig. 5A). Induction of colitis by DSS resulted in an increased MPO activity in the colon tissue compared with the normal control group (Fig. 5B). Additionally, administration of 5-FU to DSS-treated mice significantly reduced the MPO activity compared with the DSS-treated group $(\mathrm{P}<0.01$; Fig. 5C). Similarly, 5-FU + DSS-treated mice demonstrated significantly decreased $\mathrm{CD}^{+} \mathrm{T}$ cell infiltration compared with the DSS-treated group $(\mathrm{P}<0.01 ;$ Fig. $5 \mathrm{~B})$.

\section{5-FU inhibits the activation of $N F-\kappa B$ in the intestinal mucosa} of DSS mice model. NF- $\mathrm{NB}$ is a critical transcription factor in the inflammatory response. It functions as a pro-inflammatory factor and participates in the pathophysiology of intestinal inflammatory diseases (21). Previous studies 
A

CD4

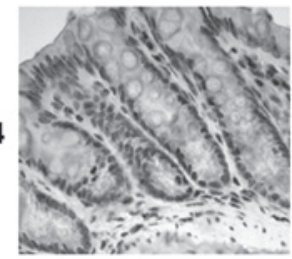

MPO

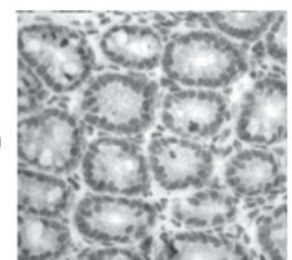

DSS

5-FU

B

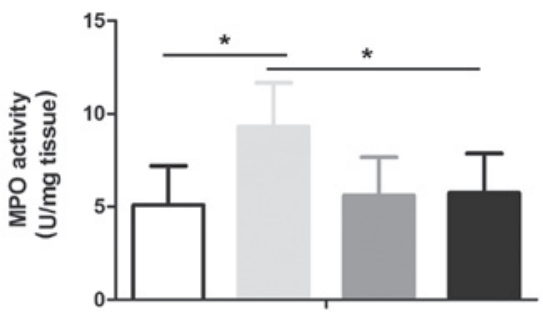

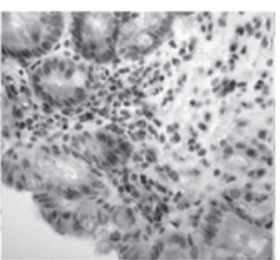

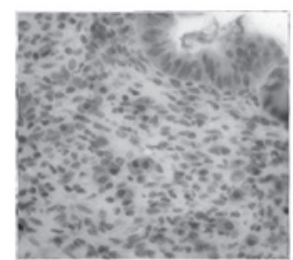

$+$

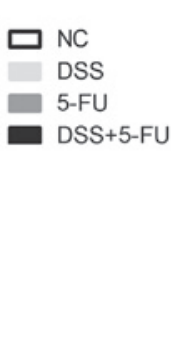

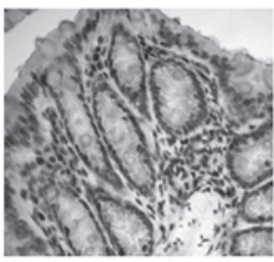
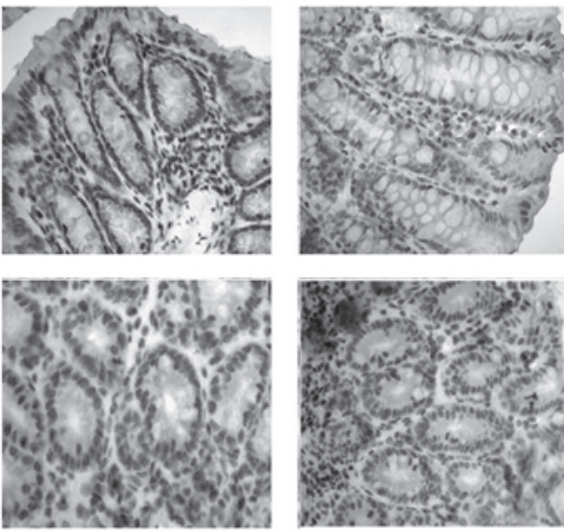

$+$

$+$

C $\quad \mathrm{Cd}^{+} \mathrm{T}$ cell

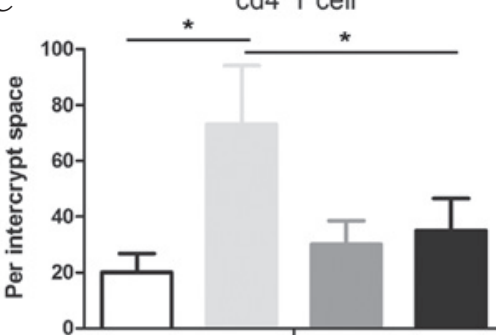

Figure 5. Effect of 5-FU treatment on the infiltration of neutrophil and CD4+ $\mathrm{T}$ cells after 7 days of treatment. (A) Microscopic appearance of representative hematoxylin and eosin, and 3,3-diaminobenzidine stained sections (magnification, x400). (B) MPO enzymatic activity as an index of neutrophil infiltration in the colon tissue and (C) $\mathrm{CD}^{+} \mathrm{T}$ cells per intercrypt space of control, DSS-treated, 5-FU-treated and DSS + 5-FU-treated mice. Data are expressed as the mean \pm standard error of the mean $(\mathrm{n}=10)$. ${ }^{*} \mathrm{P}<0.05$. MPO, myeloperoxidase; DSS, dextran sodium sulfate; 5-FU, 5-fluorouracil; NC, normal control.

A



B



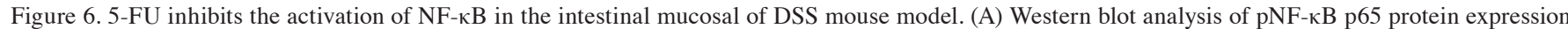
levels. (B) Fold change of pNF-кB-p65 expression levels was determined by densitometry. Data are expressed as the mean \pm standard error of the mean ( $\mathrm{n}=10$ ). ${ }^{*} \mathrm{P}<0.05$. pNF-кB, phosphorylated nuclear factor-kappa B; DSS, dextran sodium sulfate; 5-FU, 5-fluorouracil; NC, normal control.

detected activated $\mathrm{NF}-\kappa \mathrm{B}$ in colonic mucosal tissue sections of patients with IBD compared with healthy subjects $(22,23)$. Furthermore, $\mathrm{NF}-\kappa \mathrm{B}$ was identified as one of the primary factors governing the formation of the molecular network leading to various cellular functions associated with IBD (24). For example, numerous NF- $\mathrm{NB}$-dependent pro-inflammatory mediators, such as IL-1 $\beta$, TNF- $\alpha$, IL-12p40 and IL-23p19, are elevated in patients with IBD and, thus, represent therapeutic targets (25). Additionally, previous studies demonstrated that $5-\mathrm{FU}$ inhibits the activation of $\mathrm{NF}-\kappa \mathrm{B}$ and its subsequent nuclear translocation $(26,27)$. To investigate the mechanism of 5-FU's anti-inflammatory activity, the effect of 5-FU on the activation of the $\mathrm{NF}-\kappa \mathrm{B}$ pathway in intestinal epithelial cells was investigated. Changes in the expression levels of

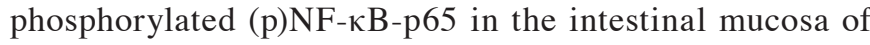
DSS-treated mice were evaluated by western blotting. As demonstrated in Fig. 6, pNF- $\mathrm{B}-\mathrm{p} 65$ was upregulated in DSS-treated mice compared with the normal control group. 5-FU treatment significantly reduced this effect compared with the DSS-treated group $(\mathrm{P}<0.05)$.

\section{Discussion}

The present study provides evidence that 5-FU modulates the immune response in acute mouse colitis by inhibition of cytokine generation, suppression of toxin-induced damage and aggravation of inflammation via reduction of the $\mathrm{NF}-\kappa \mathrm{B}$ activation. In the presence of 5-FU, DSS treatment induced 
less severe clinical symptoms of colitis, a lesser extent of epithelial damage and decreased inflammation compared with the DSS-treated mice. These results indicate that 5-FU, as an important immune-modulatory agent, participates in the epithelial response to injury and inflammation.

$5-\mathrm{FU}$ is a derivant of pyrimidine and is classified as an anti-metabolic agent. It interferes with the synthesis of DNA and RNA in healthy and tumor cells, and it is widely used in the treatment of CRC (9). Furthermore, 5-FU inhibits the synthesis of proteins, thus serving as a proteinase inhibitor, and exerts its action throughout the whole process of acute colitis (9). 5-FU decreases the synthesis and secretion of cytokines, therefore, it may alleviate the damage to mucosal tissues (12). Furthermore, Chen et al (12) demonstrated that 5 -FU modulates the pro-inflammatory cytokine response in experimental acute pancreatitis by minimizing the abnormal immune cytokines production and relieving the pathophysiological disorders. In the present study, 5-FU downregulated the serum levels of IL $1 \beta$, IFN- $\gamma$ and TNF- $\alpha$, and decreased the percentage of neutrophils in pancreatitis. An alternative study revealed that $5-\mathrm{FU}$ treatment combined with octreotide inhibited the serum levels of TNF- $\alpha$ in a mouse model of severe acute pancreatitis, which is a serious systemic inflammatory disease with a high mortality rate (28).

In the present study, increased MPO activity and $\mathrm{CD}^{+}$ expression levels were observed in the intestinal mucosa of DSS-treated mice. The decreased MPO activity that resulted from 5-FU treatment may suppress the neutrophil influx into the lamina propria and act to control disease severity through reduced secretion of pro-inflammatory cytokines, including CXCL8 and IL-17, which have been widely implicated in pathological intestinal inflammation (29).

Another effect of 5-FU was the reduction of $\mathrm{CD}^{+} \mathrm{T}$ cell accumulation in the mucosa. This may cause downregulation of pathological immune cell activation, including restraining the ability of immune cells to present antigens during colitis, a notable event previously reported during other inflammatory diseases, such as rheumatoid arthritis (30).

The present study demonstrated that $5-\mathrm{FU}$ reduced pNF- $\kappa$ B-p65 protein expression levels in DSS-induced colitis, which was in agreement with previous studies $(25,26)$. NF- $\kappa \mathrm{B}$ is an important transcription factor in the pathophysiology of several inflammatory diseases, owing to its ability to induce the expression of numerous pro-inflammatory mediators, such as cytokines, chemokines and adhesion molecules (31). A reduction in NF- $\kappa \mathrm{B}$ activation prevents the increasing production of pro-inflammatory cytokines.

Previous studies have indicated that patients administered with 5-FU may develop a certain degree of mucositis, characterized by decreased villi length and crypt cell homeostasis and accompanied with severe symptoms, including nausea and vomiting $(32,33)$. Furthermore, gastrointestinal mucositis developed in mice that received 5-FU treatment (34). However, in the current study, no intestinal mucositis was detected, possibly due to the low dose of $15 \mathrm{mg} / \mathrm{kg}$ used, which was in contrast to the dose of $400 \mathrm{mg} / \mathrm{kg}$ used by Pritchard et al (35). This dose resulted in histopathological changes to the gut, quantified as loss of crypt and villus cellularity. A previous study demonstrated that 5-FU may cause diarrhea accompanied by changes in the expression of inflammatory cytokines, including significantly increased TNF- $\alpha$, IL-1 $\beta$, IL-6, Il-17A and IL-22, throughout the entire colon of mice (36). The difference in the regulation of cytokine production may be a result of the mice species or the dose of 5-FU used in the present study.

In conclusion, the results of the current study demonstrated that low dose 5 -FU $(15 \mathrm{mg} / \mathrm{kg})$ is capable of inhibiting NF- $\kappa \mathrm{B}$ activation and effectively reducing inflammation in a mouse model of DSS-induced colitis without obvious side effects. 5-FU may therefore be a candidate therapeutic agent for the treatment of inflammatory bowel disease.

\section{References}

1. Xavier RJ and Podolsky DK: Unravelling the pathogenesis of inflammatory bowel disease. Nature 448: 427-434, 2007.

2. Ordás I, Eckmann L, Talamini M, Baumgart DC and Sandborn WJ: Ulcerative colitis. Lancet 380: 1606-1619, 2012.

3. Molodecky NA, Soon IS, Rabi DM, Ghali WA, Ferris M, Chernoff G, Benchimol EI, Panaccione R, Ghosh S, Barkema HW and Kaplan GG: Increasing incidence and prevalence of the inflammatory bowel diseases with time, based on systematic review. Gastroenterology 142: 46-54, 2012.

4. Grivennikov SI: Inflammation and colorectal cancer: colitis-associated neoplasia. Semin Immunopathol 35: 229-244, 2013.

5. Bernklev T, Jahnsen J, Lygren I, Henriksen M, Vatn M and Moum B: Health-related quality of life in patients with inflammatory bowel disease measured with the short form-36: Psychometric assessments and a comparison with general population norms. Inflamm Bowel Dis 11: 909-918, 2005.

6. Harpaz $\mathrm{N}$ and Talbot IC: Colorectal cancer in idiopathic inflammatory bowel disease. Semin Diagn Pathol 13: 339-357, 1996.

7. Sandborn WJ: Azathioprine: State of the art in inflammatory bowel disease. Scand J Gastroenterol Suppl 225: 92-99, 1998

8. Targownik LE and Bernstein CN: Infectious and malignant complications of TNF inhibitor therapy in IBD. Am J Gastroenterol 108: 1835-1842, quiz 1843, 2013.

9. Álvarez P, Marchal JA, Boulaiz H, Carrillo E, Vélez C, Rodríguez-Serrano F, Melguizo C, Prados J, Madeddu R and Aranega A: 5-Fluorouracil derivatives: A patent review. Expert Opin Ther Patents 22:107-123, 2012.

10. Bielecki K, Wiedmann M, Meyer F, Kimura W and Mössner J: Effect of 5-fluorouracil on secretion and synthesis of pancreatic digestive enzymes: Studies in isolated pancreatic acini and perfused pancreas derived from normal rats and from rats with acute necrotizing pancreatitis. Pancreas 9: 518-525, 1994.

11. Rich TA, Shepard RC and Mosley ST: Four decades of continuing innovation with fluorouracil: Current and future approaches to fluorouracil chemoradiation therapy. J Clin Oncol 22: 2214-2232, 2004.

12. Chen X-L, Ciren S-Z, Zhang H, Duan LG and Wesley AJ: Effect of 5-FU on modulation of disarrangement of immune-associated cytokines in experimental acute pancreatitis. World J Gastroenterol 15: 2032-2037, 2009

13. Luther J, Owyang SY, Takeuchi T, Cole TS, Zhang M, Liu M, Erb-Downward J, Rubenstein JH, Chen CC, Pierzchala AV, et al: Helicobacter pylori DNA decreases pro-inflammatory cytokine production by dendritic cells and attenuates dextran sodium sulphate-induced colitis. Gut 60: 1479-1486, 2011.

14. Song JL, Qian Y, Li GJ and Zhao X. Anti-inflammatory effects of kudingcha methanol extract (Ilex kudingcha C.J. Tseng) in dextran sulfate sodium-induced ulcerative colitis. Mol Med Rep 8:1256-1262, 2013.

15. Obermeier F, Dunger N, Strauch UG, Hofmann C, Bleich A, Grunwald N, Hedrich HJ, Aschenbrenner E, Schlegelberger B, Rogler G, et al: CpG motifs of bacterial DNA essentially contribute to the perpetuation of chronic intestinal inflammation. Gastroenterology 129: 913-27, 2005.

16. Livak KJ and Schmittgen TD: Analysis of relative gene expression data using real-time quantitative PCR and the 2-(Delta Delta C(T)) method. Methods 25: 402-408, 2001.

17. Bradley PP, Priebat DA, Christensen RD and Rothstein G: Cellular and extracellular myeloperoxidase in pyogenic inflammation. J Invest Dermatol 78: 206-209, 1982. 
18. Berndt BE, Zhang M, Chen GH, Huffnagle GB and Kao JY: The role of dendritic cells in the development of acute dextran sulfate sodium colitis. J Immunol 179: 6255-6262, 2007.

19. Kitajima S, Takuma S and Morimoto M: Changes in colonic mucosal permeability in mouse colitis induced with dextran sulfate sodium. Exp Anim 48: 137-143, 1999.

20. Li L, Ren F, Yun Z, An Y, Wang C and Yan X: Determination of the effects of lactoferrin in a preclinical mouse model of experimental colitis. Mol Med Rep 8:1125-1129, 2013.

21. Karrasch T and Jobin C: NF-kappaB and the intestine: Friend or foe? Inflamm Bowel Dis 14: 114-124, 2008

22. Schreiber S, Nikolaus S and Hampe J: Activation of nuclear factor kappa B inflammatory bowel disease. Gut 42: 477-484, 1998.

23. Rogler G, Brand K, Vogl D, Page S, Hofmeister R, Andus T, Knuechel R, Baeuerle PA, Schölmerich J and Gross V: Nuclear factor kappaB is activated in macrophages and epithelial cells of inflamed intestinal mucosa. Gastroenterology 115: 357-369, 1998.

24. Berndt U, Bartsch S, Philipsen L, Danese S, Wiedenmann B, Dignass AU, Hämmerle M and Sturm A: Proteomic analysis of the inflamed intestinal mucosa reveals distinctive immune response profiles in Crohn's disease and ulcerative colitis. J Immunol 179: 295-304, 2007.

25. Pizarro TT and Cominelli F: Cytokine therapy for Crohn's disease: Advances in translational research. Annu Rev Med 58 433-444, 2007.

26. Islam S, Hassan F, Tumurkhuu G, Ito H, Koide N, Mori I, Yoshida T and Yokochi T: 5-Fluorouracil prevents lipopolysaccharide-induced nitric oxide production in RAW 264.7 macrophage cells by inhibiting Akt-dependent nuclear factor-kappaB activation. Cancer Chemother Pharmacol 59: 227-233, 2007.

27. Melen-Mucha G, Balcerczak E, Mucha S, Panczyk M, Lipa S and Mirowski M: Expression of p65 gene in experimental colon cancer under the influence of 5-fluorouracil given alone and in combination with hormonal modulation. Neoplasma 51: 319-324, 2004.
28. Zhou MT, Chen BC and Sun HW: Continuous regional arterial infusion with fluorouracil and octreotide attenuates severe acute pancreatitis in a canine model. PLoS One 7: e37347, 2012.

29. Fournier BM and Parkos CA: The role of neutrophils during intestinal inflammation. Mucosal Immunol 5: 354-366, 2012.

30. Aarvak T and Natvig JB: Cell-cell interactions in synovitis: Antigen presenting cells and $\mathrm{T}$ cell interaction in rheumatoid arthritis. Arthritis Res 3: 13-17, 2001.

31. Hayden MS, West AP and Ghosh S: NF-kappaB and the immune response. Oncogene 25: 6758-6780, 2006.

32. Keefe DM, Gibson RJ and Hauer-Jensen M: Gastrointestinal mucositis. Semin Oncol Nurs 20: 38-47, 2004.

33. Azevedo OG, Oliveira RA, Oliveira BC, Zaja-Milatovic S, Araújo CV, Wong DV, Costa TB, Lucena HB, Lima RC Jr, Ribeiro RA, et al: Apolipoprotein E COG 133 mimetic peptide improves 5-fluorouracil-induced intestinal mucositis. BMC Gastroenterol 12: 35, 2012.

34. Saegusa Y, Ichikawa T, Iwai T, Goso Y, Okayasu I, Ikezawa T, Shikama N, Saigenji K and Ishihara K: Changes in the mucus barrier of the rat during 5-fluorouracil-induced gastrointestinal mucositis. Scand J Gastroenterol 43: 59-65, 2008.

35. Pritchard DM, Potten CS and Hickman JA: The relationships between $\mathrm{p} 53$-dependent apoptosis, inhibition of proliferation, and 5-fluorouracil-induced histopathology in murine intestinal epithelia. Cancer Res 58: 5453-5465, 1998.

36. Sakai H, Sagara A, Matsumoto K, Hasegawa S, Sato K, Nishizaki M, Shoji T, Horie S, Nakagawa T, Tokuyama S and Narita M: 5-Fluorouracil induces diarrhea with changes in the expression of inflammatory cytokines and aquaporins in mouse intestines. PLoS One 8: e54788, 2013. 